\title{
Short- and long-term outcomes of thoracoscopic pneumonectomy - single center experience
}

\author{
Cezary Piwkowski ${ }^{1}$, Piotr Gabryel ${ }^{1}$, Magdalena Roszak ${ }^{2}$, Bartłomiej Perek ${ }^{3}$, Mariusz Kasprzyk ${ }^{1}$ \\ ${ }^{1}$ Department of Thoracic Surgery, Poznan University of Medical Sciences, Poznan, Poland \\ 2Department of Computer Science and Statistics, Poznan University of Medical Sciences, Poznan, Poland \\ ${ }^{3}$ Department of Cardiac Surgery and Transplantology, Poznan University of Medical Sciences, Poznan, Poland
}

Videosurgery Miniinv 2021; 16 (2): 369-376

DOI: https://doi.org/10.5114/wiitm.2021.103923

\begin{abstract}
Introduction: The video-assisted thoracic surgery (VATS) approach is widely used for pulmonary lobectomy, but its application for pneumonectomy is much less common and outcomes are ambiguous.

Aim: To evaluate the feasibility and outcomes of VATS pneumonectomy.

Material and methods: This retrospective study included 19 patients with the mean age of $62.6 \pm 5.5$ years who were qualified for VATS pneumonectomy between September 1, 2010, and January 31, 2020. Indications and technical aspects were analyzed. Moreover, short- and long-term outcomes were assessed.

Results: There were no intraoperative deaths. Conversion to thoracotomy was necessary in 2 (10.5\%) patients, because of bleeding in 1 patient and technical reasons in another. One patient died during the in-hospital period due to multi-organ failure as a result of bronchopleural fistula. Five other subjects developed postoperative complications, most often atrial fibrillation $(n=3)$. One patient was readmitted for empyema of the postpneumonectomy space without bronchopleural fistula. Histopathological examination revealed that the resection was complete (RO) in all cases and the most common type of cancer was squamous cell carcinoma (79\%). Seven patients died during the follow-up: 1 because of surgical complications, 4 as a result of cancer progression, and 2 for non-cancer related reasons. Median survival was 47 months. One- and five-year probability of survival estimated by means of the Kaplan-Meier method was $0.88 \pm 0.07$ and $0.43 \pm 0.15$, respectively.

Conclusions: VATS pneumonectomy can be performed safely, without increased risk of intraoperative and postoperative complications. It enables a complete lung cancer resection and is likely to provide good short- and long-term outcomes.
\end{abstract}

Key words: thoracoscopy/video-assisted thoracic surgery, lung cancer, minimally invasive surgery, pneumonectomy.

\section{Introduction}

Anatomical lung resection (lobectomy and segmentectomy) is the mainstay treatment for early-stage non-small cell lung cancer (NSCLC) [1]. Owing to several advantages, such as less surgical trauma, a lower complication rate and improved postoperative rehabilitation, a majority of the aforementioned operations are currently performed through the video-assisted thoracic surgery (VATS) approach [2, 3]. Pneumonectomy, as a significantly more extensive procedure, is applied in advanced cases of NSCLC, including centrally located tumors invading large vessels or the main bronchus. Due to this reason, operations are usually carried out through the conventional thoracotomy approach. Of note, in carefully selected

\section{Address for correspondence}

Piotr Gabryel MD, PhD, Department of Thoracic Surgery, Poznan University of Medical Sciences, Poznan, Poland, phone: +48 603444042 , e-mail: piotrgabryel@gmail.com 
cases, the minimally invasive approach for pneumonectomy may be an intriguing option [4]. However, contrary to VATS lobectomy or segmentectomy, minimally invasive pneumonectomy is not a routine procedure; thus clinical studies dealing with particularly long-term outcomes should be widespread among the thoracic surgical community.

\section{Aim}

The aim of this study was to analyze the feasibility of VATS pneumonectomy, including the qualification criteria and surgical technique, as well as to evaluate the short- and long-term outcomes of VATS pneumonectomies performed in a single thoracic surgical center.

\section{Material and methods}

The Local Bioethical Committee waived the need for ethics approval and the need to obtain written consent for the collection, analysis and publication of the retrospectively collected and anonymized data for this non-interventional study.

\section{Demographics, preoperative evaluation, and qualification for surgery}

The medical records of all patients who underwent lung anatomical resection from September 1, 2010, to January 31, 2020 were reviewed. The exclusion criteria were: planned open approach (thoracotomy) and extent of resection lesser than pneumonectomy (lobectomy, sublobar resection). All patients who underwent VATS pneumonectomy were included.

During preoperative assessment, all patients underwent a detailed interview, physical examination, chest computed tomography (CT) scan, abdominal ultrasound, electrocardiography, pulmonary function tests (PFTs), and fiberoptic bronchoscopy. If indicated, echocardiography, exercise tests, diffusion lung capacity for carbon monoxide (DLCO), and positron emission tomography-computed tomography (PET$\mathrm{CT}$ ) were performed. Perioperative risk was assessed according to the American Society of Anesthesiologists (ASA) scale, the Thoracic Revised Cardiac Risk Index (ThRCRI) and European Risk Models for Morbidity (EuroLung1) and Mortality (EuroLung2). The predicted postoperative pulmonary function was calculated on the basis of PFTs (FEV 1 and FVC) after considering the extent of lung parenchyma involve- ment by the tumor as well as the bronchial obturation observed in fiberoptic bronchoscopy.

Patients qualified for VATS pneumonectomy only when less extensive (sleeve) resection was not possible and it was certain that VATS could be performed safely, allowing for complete (RO) resection. The detailed qualification criteria were as follows:

1. Invasion of the distal part of the main bronchus or pulmonary artery when sleeve resection was not possible.

2. Invasion of two (left-sided) or three (right-sided) lobes.

3. Tumor with metastasis to the other lobe of the same lung after exclusion of distant metastases in PET-CT, when less extensive resection (lobectomy with wedge resection or Nd:YAG laser resection) was not possible.

Patients did not qualify for VATS pneumonectomy in the following situations:

1. Locally advanced tumor with extensive infiltration of the pulmonary vessels, chest wall, or mediastinum.

2. Tumor size $>6-8 \mathrm{~cm}$.

3. When it was not possible to exclude the option of sleeve resection.

4. After neoadjuvant/induction therapy (e.g., chemotherapy and/or radiotherapy).

\section{Anesthesia, surgical approach, and technique}

Surgery was performed under general anesthesia with double-lumen intubation and single lung ventilation. Patients were placed in the lateral decubitus position with flexion of the surgical table at the level of the scapula tip. Two types of VATS approach were applied: 3-portal VATS (3P-VATS) and uniportal VATS (U-VATS). In the case of 3P-VATS, a $5 \mathrm{~cm}$ long utility incision in the $4^{\text {th }}$ or $5^{\text {th }}$ intercostal space anterior to the latissimus dorsi muscle was performed, and two ports were placed in the $7^{\text {th }}$ intercostal space of the anterior axillary line and in the $8^{\text {th }}$ intercostal space of the posterior axillary line. In the case of U-VATS, only a $5 \mathrm{~cm}$ long utility incision was made in the $4^{\text {th }}$ or $5^{\text {th }}$ intercostal space. No rib spreader was used. However, in U-VATS, an Alexis wound protector/retractor (Applied Medical, Rancho Santa Margarita, CA) was placed for utility incision. A $30^{\circ}$ camera was introduced through the anterior port in the case of 3P-VATS or through the posterior portion of the utility incision in U-VATS. An endoscopic dissector, endoscopic hook, and LigaSure 
(Medtronic, Dublin, Ireland) were used for soft tissue dissection and lymphadenectomy. The pulmonary vessels and bronchus were sutured and divided with EndoGia endostaplers (Medtronic, Dublin, Ireland): the veins and arteries with vascular (white or gold) and the bronchus with violet reloads. The typical steps involved in the surgery were as follows:

1. On the right side, dissection of the pulmonary ligament and posterior mediastinal pleura; stations 9, 8, and 7 lymph nodes; upper pulmonary vein; right pulmonary artery; lower pulmonary vein; stations 10, 4, and 2 lymph nodes; and the main bronchus was performed.

2. On the left side, the dissection of the upper pulmonary vein; stations 5 and 6 lymph nodes; left pulmonary artery; lower pulmonary vein; stations 7,8 , and 4 lymph nodes; and the main bronchus was performed.

The lung was removed from the pleural cavity in an endobag. The bronchial stump was submerged under water and checked for any air leak. The bronchial stump was not covered with tissue flaps. If an epidural anesthesia catheter was not placed in the patient before surgery, paravertebral block with bupivacaine was performed at this time point. One 28-F chest tube was inserted through the anterior port (3P-VATS) or the posterior part of the utility incision (U-VATS).

After surgery, all patients were initially admitted to the Intensive Care Unit and, subsequently, transferred to the Thoracic Surgery Department. The patients were released from the hospital if there were no complications and the follow-up fiberoptic bronchoscopy was normal. Short- and long-term results, including probability of survival according to the Kaplan-Meier method, were assessed.

\section{Data presentation}

Normally distributed continuous variables are presented as mean with standard deviation (mean \pm SD) whereas the others are presented as median with the range (minimum-maximum). Categorical data are expressed as numbers $(n)$ with percentages (\%). Probability of survival was assessed according to the Kaplan-Meier method.

\section{Results}

From a total of 324 patients who underwent pneumonectomy between September 1, 2010, and
January 31, 2020, 19 (5.9\%) patients were qualified for VATS pneumonectomy. The study group comprised 15 (78.9\%) men and 4 (21.1\%) women, mean age of $62.6 \pm 5.5$ years (range: $50-73$ years). Data on the demographics are presented in Table I.

Seventeen (89.5\%) patients were operated on by 3-portal and 2 (10.5\%) patients by uniportal VATS. Conversion to anterolateral thoracotomy was necessary in 2 (10.5\%) patients.

\section{Patients with VATS pneumonectomy converted to thoracotomy}

In the first case, the patient with class 3 obesity $\left(\mathrm{BMI}>40 \mathrm{~kg} / \mathrm{m}^{2}\right)$ and a large $(7.1 \mathrm{~cm}$ diameter) right lung tumor was qualified for 3-portal VATS pneumonectomy. Because of the high position of the diaphragm, tumor dimension, and the partially expanded lung, the space in the pleural cavity was extremely limited. During dissection of the soft tissues in the area of A1/A3 arteries and the azygos vein, dark blood bleeding occurred. Because of difficult anatomical conditions, determination of the bleeding source was not possible. The bleeding area

Table I. Patient demographics $(n=19)$

\begin{tabular}{|c|c|}
\hline Characteristic & Value \\
\hline Age [years] ${ }^{a}$ & $62.6 \pm 5.5(50-73)$ \\
\hline \multicolumn{2}{|l|}{ Sex, $n(\%):$} \\
\hline Male & $15(78.9)$ \\
\hline Female & $4(21.1)$ \\
\hline \multicolumn{2}{|l|}{ Comorbidities, $n(\%)$ : } \\
\hline Hypertension & $9(47.4)$ \\
\hline Chronic obstructive pulmonary disease & $9(47.4)$ \\
\hline Diabetes mellitus & $4(21.0)$ \\
\hline Coronary heart disease & $2(10.5)$ \\
\hline \multicolumn{2}{|l|}{ Pulmonary function tests ${ }^{a}$ : } \\
\hline $\mathrm{FEV}_{1} \%$ & $77.9 \pm 22.6 \%$ \\
\hline FVC\% & $86.2 \pm 24.9 \%$ \\
\hline \multicolumn{2}{|l|}{ ASA scale, $n(\%):$} \\
\hline 2 & $9(47.4)$ \\
\hline 3 & $10(63.6)$ \\
\hline \multicolumn{2}{|l|}{ ThRCRI, $n$ (\%): } \\
\hline A & $17(89.5)$ \\
\hline $\mathrm{B}$ & $2(10.5)$ \\
\hline
\end{tabular}

$F E V_{1}$ - forced expiratory volume in 1 s, FVC - forced vital capacity, ASA - American Society of Anesthesiologists, ThRCRI - Thoracic Revised Cardiac Risk Index. ${ }^{a}$ Data are expressed as mean \pm standard deviation (range). 
was compressed, thoracotomy was performed, and the azygos vein injury was located and sutured. The remaining part of the surgery was uneventful. The surgery time was $105 \mathrm{~min}$, and the estimated blood loss was $800 \mathrm{ml}$. Blood transfusion was not deemed necessary. The postoperative course was uneventful, the chest tube was removed on POD 3, and the patient was discharged on POD 8.

In the second case, the patient qualified for left 3-portal VATS pneumonectomy because of squamous-cell carcinoma infiltrating the upper and lower lobar bronchi. A major part of the surgery was uneventful. However, after division of the main bronchus with the endostapler, the suction catheter tip was

Table II. Indications for VATS pneumonectomy

\begin{tabular}{|lc|}
\hline Indication $^{\text {a }}$ & Value \\
\hline Infiltration of distal part of main bronchus & $12(63.1)$ \\
\hline $\begin{array}{l}\text { Peripheral infiltration of two or three lobes of } \\
\text { the lung }\end{array}$ & $6(31.6)$ \\
\hline $\begin{array}{l}\text { Tumor with metastasis to other lobe not } \\
\text { amenable for less extensive resection }\end{array}$ & $1(5.3)$ \\
${ }^{a}$ Data are expressed as number (\%).
\end{tabular}

Table III. Results of histopathological examination

\begin{tabular}{|c|c|}
\hline Characteristic & Value \\
\hline \multicolumn{2}{|l|}{ Histology, $n$ (\%): } \\
\hline Squamous cell carcinoma & $15(79.0)$ \\
\hline Large-cell carcinoma & $2(10.5)$ \\
\hline Adenocarcinoma & $2(10.5)$ \\
\hline Maximal tumor diameter $[\mathrm{mm}]^{\mathrm{a}}$ & $42 \pm 12(29-71)$ \\
\hline \multicolumn{2}{|l|}{ Clinical stage, $n(\%)$ : } \\
\hline । & $2(11.1)$ \\
\hline II & $9(47.5)$ \\
\hline III & $8(44.4)$ \\
\hline \multicolumn{2}{|l|}{ Lymph node metastases, $n$ (\%): } \\
\hline NO & $6(33.3)$ \\
\hline N1 & $9(47.5)$ \\
\hline N2 & $4(22.2)$ \\
\hline \multicolumn{2}{|l|}{ Bronchial margin width in NSCLC [mm] } \\
\hline All patients & $11.4 \pm 4.2(4-20)$ \\
\hline Non-converted VATS $(n=17)$ & $11.9 \pm 4.1(4-20)$ \\
\hline Conversion to thoracotomy $(n=2)$ & $7.5 \pm 2.5(5-10)$ \\
\hline Complete resection (RO) in NSCLC, $n(\%)$ & 19 (100) \\
\hline
\end{tabular}

found to be embedded in the bronchial suture line. Thoracotomy was performed, the bronchial stump was shortened, and the catheter was removed, followed by closing the bronchus with a running suture. The surgery time was $100 \mathrm{~min}$, and the estimated blood loss was $100 \mathrm{ml}$. The postoperative course was uneventful, the chest tube was removed on POD 5 , and the patient was discharged on POD 10.

\section{Patients with pneumonectomies completed by VATS}

Among the group of 17 patients in whom surgery was completed by VATS, the mean surgery time was $121 \mathrm{~min}$ (range: 85-200 $\mathrm{min}$ ) and the median estimated blood loss was $147 \mathrm{ml}$ (50-300 ml). The median chest drainage time was 3 days (range: 2-4 days), and the median hospital stay was 8 days (range: 5-90 days). Postoperative complications occurred in $6(35.3 \%)$ of the 17 patients in this group: 1. Atrial fibrillation in 2 patients.

2. Respiratory failure, sepsis and multi-organ dysfunction syndrome in 1 patient.

3. Esophageal perforation which required re-thoracotomy with suturing of the perforation on POD 6, and subsequent omentopexy, gastrostomy, and open thoracostomy on POD 15 because of recurrence.

4. Atrial fibrillation and subsequent superior mesenteric artery embolism on POD 4 in 1 patient, which required small intestine resection and total parenteral nutrition.

5. Bronchopleural fistula diagnosed on POD 5 in 1 patient. Despite chest tube placement, the patient's condition deteriorated rapidly, with sepsis, multi-organ dysfunction and death on POD 5 . In this patient, during the surgery, a small air leak was noted from the bronchial stump. The stump was then additionally sutured with a running suture and confirmed to be airtight.

One patient required readmission because of empyema of the postpneumonectomy space without bronchopleural fistula. Treatment with chest drainage and antibiotics was successful (Table II).

\section{Results of histopathological examination}

Squamous cell carcinoma was the most common type of NSCLC and was diagnosed in 15 (79.0\%) patients. Most patients were in clinical stage II or III according to the $8^{\text {th }}$ TNM classification (Table III).

Resection was complete $\left(R_{0}\right)$ in all patients with NSCLC, both completed by VATS and converted to 
thoracotomy. The mean distance from the tumor to the bronchial suture line was $11.4 \pm 4.2 \mathrm{~mm}$, range: 4-20 $\mathrm{mm}$. In 1 patient with a $4 \mathrm{~mm}$ margin, dysplastic epithelial cells were found in the suture line; however, at 33 months no local recurrence was observed.

\section{Adjuvant treatment and follow-up}

A total of twelve of 19 (63.2\%) patients received adjuvant treatment, of whom ten received chemotherapy and two received chemo- and radiotherapy.

The median follow-up time was 882 days (range: 105-2614 days). Progression of the disease was noted in 4 patients, with distant metastases in 1 , chest wall metastases in another 1 , and mediastinal recurrence in 2 patients. The mean time to progression was 529 days (range: 300-806 days). A total of 7 patients died during the follow-up: 1 because of surgical complications, 4 as a result of cancer progression, and 2 for reasons not related to cancer and its treatment. Median survival was 74 months (interquartile range $(I Q R)=49-106$ months). One-, two- and 5-year probability of survival estimated by means of the Kaplan-Meier method was $0.88 \pm 0.07$, $0.82 \pm 0.09$ and $0.43 \pm 0.15$, respectively (Figure 1 ). Estimated 1-, 2- and 5-year freedom from lung cancer recurrence after VATS pneumonectomy was 0.87 $\pm 0.09,0.79 \pm 0.11$ and $0.71 \pm 0.13$, respectively (Figure 2).

\section{Discussion}

Pneumonectomy is one of the most extensive operations performed by thoracic surgeons. It is associated with higher incidence of complications compared with lesser resections and can significantly impair a patient's fitness and quality of life, both in short- and long-term perspectives. Pneumonectomy is generally performed through an open approach, usually by antero-lateral or postero-lateral thoracotomy. However, with the development of minimally invasive thoracic surgery, such operations are now even conducted through the minimally invasive approach. The VATS approach was introduced to thoracic surgery in the 1990s. Initially, it was used for less extensive procedures, but it was gradually adopted for anatomical lung resections $[5,6]$. VATS lobectomy was proved to be associated with less surgical trauma, decreased postoperative pain, a lower complication rate, especially in terms of pulmonary complications (e.g., atelectasis and pneumonia), im- proved postoperative rehabilitation, shorter hospital stay, and better quality of life compared with the thoracotomy approach $[7,8]$. Despite the fact that in many countries, the cost of VATS anatomical lung resection far exceeds the cost of thoracotomy, the former became the preferred approach in major thoracic surgery centers worldwide because of its evident clinical benefits $[9,10]$.

The rapid development of minimally invasive thoracic surgery did not, however, involve VATS pneumonectomy. Despite the fact that the first few VATS pneumonectomies were performed in the early 1990s, the number of procedures performed worldwide remained low [11]. Our experience was

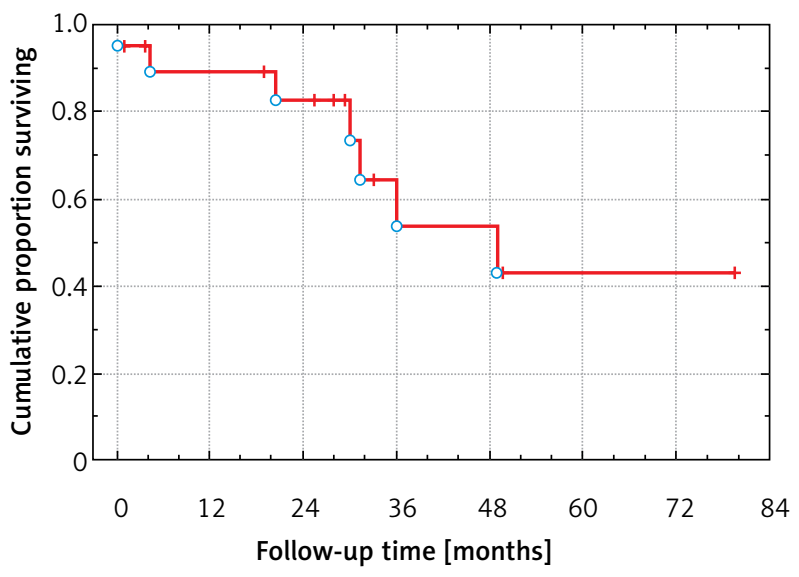

Probability of survival $\circ$ Complete + Censored

Figure 1. One-, two- and five-year probability of survival after VATS pneumonectomy

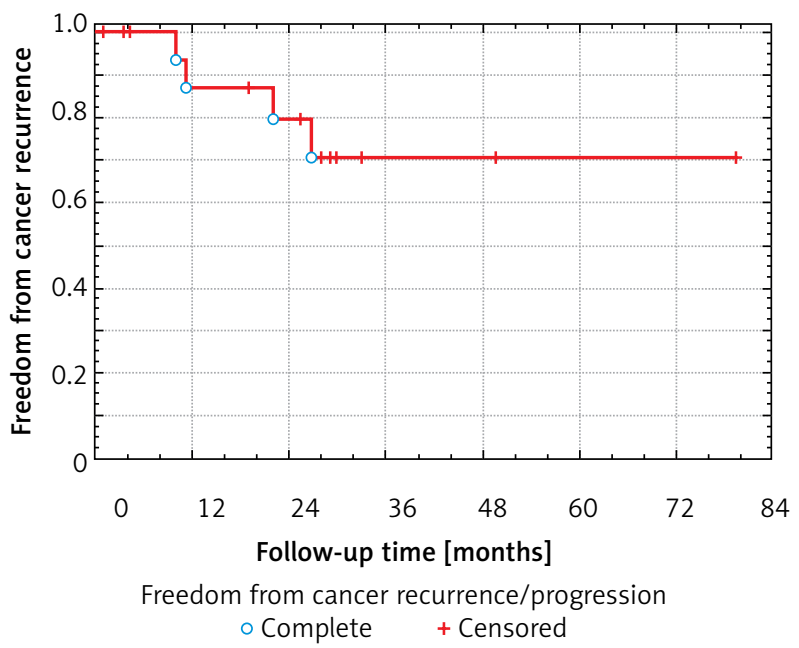

Figure 2. Estimated one-, two- and five-year freedom from lung cancer recurrence after VATS pneumonectomy 
also similar. Although a minimally invasive surgery program was started at our center in 1999 and the total number of VATS lobectomies performed for oncological and other indications exceeded 1000, the numbers of attempted VATS pneumonectomies is low. The most important reason for this is probably the fact that the majority of the patients are qualified for pneumonectomy because of more locally advanced tumors and usually larger, invading anatomical structures of the pulmonary hilum (pulmonary artery and vein and main bronchus) or the chest wall [12]. Situations in which the patient can be qualified for the VATS approach (e.g., smaller tumors invading the distal part of the main bronchus, peripheral infiltration of two or three lobes of the lung or peripheral tumor with hilar lymph node metastasis, and tumor with metastasis to other lobes not amenable for less extensive resection) are much less common. Therefore, only a very selected group of patients is eligible to undergo VATS.

It must be emphasized that during the process of qualification for surgery, the possibility of performing sleeve resection should always be excluded. It has been proven that sleeve lobectomy is superior to pneumonectomy in terms of postoperative complications and the quality of life and provides comparable long-term results $[13,14]$. Although cases of VATS sleeve lobectomies have been described, these operations are extremely demanding from the surgical perspective and rarely performed $[15,16]$. Hence, if it is not possible to unequivocally exclude sleeve lobectomy based on the results of preoperative studies, thoracotomy should be considered.

The preferred surgical approach in the case of VATS pneumonectomy is similar to that in the case of VATS lobectomy and so is the mode of division of the pulmonary vessels. Because of the potentially catastrophic consequences of eventual pulmonary artery injury, particular care must be taken during its dissection and division. In the case of centrally located tumors, the mobility of the hilum is limited and the length of the vessel for stapler application is short; therefore, the traction must be performed very carefully. Bleeding from the vessel staple line may sometimes occur, but it is rare and usually self-limiting. If there are any doubts regarding the quality of staplers, a vascular clamp can be applied proximally around the artery before stapler placement [17]. Another useful technique, described in the case of right U-VATS with a large artery, is the separate division of the Boyden trunk first and the intermediate artery afterwards [18]. On the left side, prior dissection of the lymph nodes of the aorto-pulmonary window facilitates left main pulmonary dissection and division.

Division of the main bronchus is the last step during VATS pneumonectomy. It is of utmost importance to obtain sufficient distance between the tumor and bronchial suture line in case of main bronchus invasion so as to achieve complete resection (RO). Our results indicated that particular attention should be paid regarding this aspect in patients operated on the right side because of the shorter length of the right main bronchus, as well as in patients with more proximal tumor invasion. Because the intraoperative manual examination is virtually impossible during VATS, preoperative fiberoptic bronchoscopy by the operating surgeon and intraoperative frozen section of the resected bronchial line are mandatory. Another important aspect is to obtain the shortest possible bronchial stump in order to diminish the risk of bronchopleural fistula. In this aspect, the right side does not usually pose any particular problem. On the left side, however, it may be more difficult because of the location of the left main bronchus under the aortic arch. Applying more traction to the bronchus can help obtain a short bronchial stump $[17,19]$.

The issue of conversion to thoracotomy in the case of VATS pneumonectomy has been relatively poorly researched. We adopted the division of causes of conversion into three groups: "oncologic", "technical", and "complications" [20]. In this study, the conversion was necessary in 2 patients owing to complications (bleeding) and technical reasons (suction catheter tip in the bronchial suture line). Despite increased intraoperative blood loss in the first patient, the postoperative period for both patients was uncomplicated and the fact of conversion did not lead to an increased postoperative complication rate. The conversion rate of VATS pneumonectomy reported in the literature is varied and falls between $2 \%$ and $25 \%$ [21]. It must be stressed that except for emergent indications, the conversion has not been proven to be related to the postoperative complication rate and thus should be not considered as an indicator of the surgery quality [22].

The complication rate after pneumonectomy seems to be similar after VATS and thoracotomy [23]. Although it has been observed that the postoperative pain was lower after VATS pneumonectomy 
as compared with that after thoracotomy, it did not translate into lower incidence of pulmonary complications [24]. In our case series, most of the complications were not directly related to the minimally invasive approach. In only 2 patients, the cause of complication was strictly surgical. Esophageal perforation, due to its delayed occurrence, could have been caused by thermal injury resulting from the use of monopolar electrocautery during dissection of the subcarinal and paraoesophageal lymph nodes. The use of high-energy devices, which were unavailable at that time, could have presumably prevented esophageal injury. Bronchopleural fistula occurred in a patient operated on the left side, in whom the bronchial stump air leak was detected after division of the bronchus during surgery. After applying an additional running suture, the stump was airtight. Because the stump was short and located deeply under the aortic arch as well as surrounded by the neighboring tissues, covering it with a pedicled tissue flap was not considered necessary. It is uncertain, but not likely, that this maneuver would have prevented bronchopleural fistula.

Most of the studies published so far have focused on early VATS pneumonectomy results, while only a few have analyzed long-term outcomes. In our study, despite the limited number of patients, longterm outcomes seem to be good. Only 4 patients out of 19 had recurrence of cancer during follow-up, which presumably resulted from the complete resection (RO) obtained in all cases and at the same time confirmed the oncological effectiveness of VATS pneumonectomy. Median survival was 74 months, and $43 \%$ of patients survived 5 years after surgery. Comparable results were obtained by Battoo et al. - median survival in stage I-II was 80 months and in stage III-IV was 42 months [25]. In a multi-center study conducted by Yang et al. which included the largest group of patients so far (359 patients after pneumonectomy, including 124 operated on by the thoracoscopic approach), 5-year survival after VATS pneumonectomy was $47 \%$. It is worth mentioning that the authors found no significant differences in overall survival between the open and VATS group [26]. The results of all those studies suggest that VATS pneumonectomy allows one to achieve good outcomes of treatment.

The most important limitation of the study was the relatively low number of patients included. This was due mainly to two reasons: first, the number of pneumonectomies performed in NSCLC is low and is still decreasing; secondly, the criteria for qualifying for VATS pneumonectomy are very narrow. Another limitation was the lack of comparison of VATS to a thoracotomy group. Both problems could probably be solved by conducting a retrospective, multicenter study with propensity score matching of VATS and thoracotomy patients. A prospective study with randomization of patients is unlikely to be feasible due to the long time that would be necessary to gather the appropriate group of patients.

\section{Conclusions}

VATS pneumonectomy can be performed safely, without increased risk of intraoperative and postoperative complications, allows one to obtain a complete resection in NSCLC and is likely to provide good short- and long-term results.

\section{Acknowledgments}

The authors would like to thank Enago (www. enago.com) for the English language review.

\section{Conflict of interest}

The authors declare no conflict of interest.

\section{References}

1. Shin JY, Yoon JK, Marwaha G. Progress in the treatment and outcomes for early-stage non-small cell lung cancer. Lung 2018; 196: 351-8.

2. Al-Ameri M, Bergman P, Franco-Cereceda A, et al. Video-assisted thoracoscopic versus open thoracotomy lobectomy: a Swedish nationwide cohort study. J Thorac Dis 2018; 10: 3499-506.

3. Dziedzic R, Marjanski T, Binczyk F, et al. Favourable outcomes in patients with early-stage non-small-cell lung cancer operated on by video-assisted thoracoscopic surgery: a propensity score-matched analysis. Eur J Cardiothorac Surg 2018; 54 : 547-53.

4. Piwkowski C, Gabryel P, Kasprzyk M, et al. Video-assisted thoracic surgery pneumonectomy: the first case report in Poland. Videosurgery Miniinv 2012; 7: 197-201.

5. McKenna RJ, Houck W, Fuller CB. Video-assisted thoracic surgery lobectomy: experience with 1,100 cases. Ann Thorac Surg 2006; 81: 421-6.

6. Zhong L, Hu W, Li S, et al. Clinical study of video-assisted thoracoscopic surgery wedge resection in early-stage lung cancer by tumor mapping with indocyanine green. Videosurgery Miniinv 2019; 14: 545-50.

7. Bendixen M, Jørgensen OD, Kronborg C, et al. Postoperative pain and quality of life after lobectomy via video-assisted thoracoscopic surgery or anterolateral thoracotomy for early stage 
lung cancer: a randomised controlled trial. Lancet Oncol 2016; 17: 836-44

8. Ye B, Wang M. Video-assisted thoracoscopic surgery versus thoracotomy for non-small cell lung cancer: a meta-analysis. Comb Chem High Throughput Screen 2019; 22: 187-93.

9. Piwkowski C, Gabryel P, Galecki B, et al. High costs as a slow down factor of thoracoscopic lobectomy development in Poland - an institutional experience. Videosurgery Miniinv 2013; 8: 334-41.

10. Alpay L, Lacin T, Teker D, et al. A comparative cost analysis study of lobectomy performed via video-assisted thoracic surgery versus horacotomy in Turkey. Videosurgery Miniinv 2014; 9: 409-14.

11. Roviaro G, Varoli F, Rebuffat C, et al. Major pulmonary resections: pneumonectomies and lobectomies. Ann Thorac Surg 1993; 56: 779-83.

12. Gibas A, Chwirot P, Rzyman W. Results of pneumonectomy in non-small cell lung cancer patients. Kardiochir Torakochir Pol 2009; 6: 142-8.

13. Pages PB, Mordant P, Renaud S, et al. Sleeve lobectomy may provide better outcomes than pneumonectomy for non-small cell lung cancer. A decade in a nationwide study. I Thorac Cardiovasc Surg 2017; 153: 184-95.e3.

14. Kasprzyk M, Dyszkiewicz W, Piwkowski C, et al. Sleeve lobectomy as an alternative to pneumonectomy in patients with operable lung cancer. Neoplasma 2013; 60: 62-7.

15. Huang J, Li S, Hao Z, al. Complete video-assisted thoracoscopic surgery (VATS) bronchial sleeve lobectomy. J Thorac Dis 2016; 8: 553-74.

16. Kocher GJ. Multiport and uniportal VATS sleeve resections. J Thorac Dis 2019; 11: 1091-3.

17. Craig SR, Walker WS. Initial experience of video assisted thoracoscopic pneumonectomy. Thorax 1995; 50: 392-5.

18. Gonzalez-Rivas D, Delgado M, Fieira E, et al. Uniportal video-assisted thoracoscopic pneumonectomy. J Thorac Dis 2013; 5: S246-52.

19. Sahai RK, Nwogu CE, Yendamuri S, et al. Is thoracoscopic pneumonectomy safe? Ann Thorac Surg 2009; 88: 1086-92.

20. Decaluwe H, Petersen RH, Hansen H, et al. Major intraoperative complications during video-assisted thoracoscopic anatomical lung resections: an intention-to-treat analysis. Eur J Cardiothorac Surg 2015; 48: 588-99.

21. Nagai S, Imanishi N, Matsuoka T, et al. Video-assisted thoracoscopic pneumonectomy: retrospective outcome analysis of 47 consecutive patients. Ann Thorac Surg 2014; 97: 1908-13.

22. Nwogu CE, Yendamuri S, Demmy TL. Does thoracoscopic pneumonectomy for lung cancer affect survival? Ann Thorac Surg 2010; 89: S2102-6.

23. Liu Y, Gao Y, Zhang H, et al. Video-assisted versus conventional thoracotomy pneumonectomy: a comparison of perioperative outcomes and short-term measures of convalescence. J Thorac Dis 2016; 8: 3537-42.

24. Jeon JH, Kang CH, Kim HS, et al. Video-assisted thoracoscopic lobectomy in non-small-cell lung cancer patients with chronic obstructive pulmonary disease is associated with lower pulmonary complications than open lobectomy: a propensity scorematched analysis. Eur J Cardiothorac Surg 2014; 45: 640-5.
25. Battoo A, Jahan A, Yang Z, et al. Thoracoscopic pneumonectomy: an 11-year experience. Chest 2014; 146: 1300-9.

26. Yang CJ, Yendamuri S, Mayne NR, et al. The role of thoracoscopic pneumonectomy in the management of non-small cell lung cancer: a multicenter study. J Thorac Cardiovasc Surg 2019; 158: 252-64.

Received: 20.07.2020, accepted: 12.09.2020. 